\title{
Improved Signal-to-Noise Ratio (SNR), Bandwidth-Enhanced Electrically Small Antenna Augmented with Internal Non-Foster Elements
}

\author{
Ting Shi, Student Member, IEEE, Ming-Chun Tang, Senior Member, IEEE, Zhentian Wu, Student \\ Member, IEEE, Hexiu Xu, Senior Member, IEEE, and Richard W. Ziolkowski, Fellow, IEEE
}

\begin{abstract}
Non-Foster technology facilitates the ability to surpass the Chu bandwidth limit associated with electrically small antennas (ESAs). Nonetheless, in addition to challenging stability issues, the enhanced performance can come at the cost of increased noise and resistance losses generated by the active circuit. Consequently, low total efficiency and degraded signal-to-noise ratio (SNR) values can arise. Stability and SNR have dominated most reports to date; little has been discussed about the underlying innovative physics of non-Foster augmented radiators. In this communication, we propose a broad bandwidth non-Foster ESA, emphasizing those aspects. By embedding a non-Foster element into the near-field resonant parasitic (NFRP) element of a metamaterial-inspired antenna, its electrically small size is maintained. On the other hand, a 5-times enhancement of its -10-dB fractional bandwidth (15-times its -3-dB bandwidth) is measured, significantly surpassing its passive Chu limit. Under good matching, the measurements demonstrate that this non-Foster ESA achieves a $1.05 \mathrm{dBi}$ peak gain, and realizes average 5.0 dB SNR and 17 dB gain improvements over its passive counterpart.
\end{abstract}

Index Terms-Electrically small antenna, non-Foster circuits, radiation pattern, signal-to-noise ratio

\section{INTRODUCTION}

Electrically small antennas (ESAs) with broadband performance are essential for the development of miniaturized, broadband communication systems for future fifth generation $(5 \mathrm{G})$ applications. It is historically known that the physics and engineering associated with ESAs are difficult. Their compact sizes inevitably lead to contradictions between simultaneous bandwidth, efficiency, and directivity performance characteristics $[1,2]$. They also naturally lead

Manuscript received on 09 Nov., 2018; revised on 11 Jan., 2019; and accepted on 14 Jan, 2019.

This work was supported in part by the National Natural Science Foundation of China, contract number 61471072; in part by Graduate Scientific Research and Innovation Foundation of Chongqing, China, contract number CYB18069; in part by the Funding of the leading research talent cultivation plan of Chongqing University, contract number cqu2017hbrc1A08; in part by the Fundamental Research Funds for the Central Universities, contract number 2018CDQYTX0025; in part by the Funding of the Innovative Leading Talents in Science and Technology of Chongqing, contract number CSTCCXLJRC201705; in part by Funding of the Young Backbone Teachers in Colleges and Universities of Chongqing, contract number 0307001104102; and in part by the Australian Research Council grant number DP160102219.

T. Shi, M. -C. Tang, and Z. Wu are with the Key Laboratory of Dependable Service Computing in Cyber Physical Society Ministry of Education, College of Communication Engineering, Chongqing University, Chongqing 400044, China (E-mail: tangmingchun@cqu.edu.cn);

$\mathrm{H}$. $\mathrm{Xu}$ is with the RF and Microwave Labs, Air Force Engineering University, Xi'an, 710051, China.

R. W. Ziolkowski is with the University of Technology Sydney, Global Big Data Technologies Centre, Ultimo NSW 2007, Australia (E-mail: Richard.Ziolkowski@uts. edu.au). to small radiation resistances and large reactance values, making them difficult to match to industry standard $50 \Omega$ sources. While passive matching networks can be employed to attain impedance matching, the maximum achievable bandwidth is inevitably bounded by the Bode-Fano limits [3]. Moreover, the Wheeler-Chu theoretical limits set further bounds on the size, bandwidth, and efficiency even when ESAs are well-matched [4, 5]. As summarized in [6], passive ESAs reported over the past sixty years have been severely limited by the Wheeler-Chu limit.

Non-Foster elements, which do not obey the Foster reactance theorem for passive, lossless reactive elements [7], facilitate an effective method to overcome these limits. They are generally conceived as negative capacitors or inductors and are very often realized with negative impedance converters (NICs) [8]. However, they also introduce an inevitable increase in noise and resistance losses, which possibly result in lowered total efficiencies and degraded signal-to-noise ratio (SNR) values. The combination of these features can especially impact a receiving antenna system. The potential deterioration of the SNR beyond an acceptable detection limit gives grave concerns for the degradation of communications reliability.

While many of the originally reported non-Foster ESAs relied simply on simulated analyses of ideal models with few tested prototypes [9-13], several works have reported experimental verifications of the enhanced bandwidth properties [14-18]. Nevertheless, while higher gain and lower noise values are essential for SNR improvements, very few reports have included the analysis and experimental investigations of gain, noise, and SNR issues. A measured $9 \mathrm{~dB}$ SNR improvement was realized at $30 \mathrm{MHz}$ in [19]. In contrast, it was demonstrated in [20] that a non-Foster matched antenna does not provide any SNR improvement compared with its passive version. It was concluded in [21] that the associated increase in noise may be an acceptable tradeoff for bandwidth. These conflicting conclusions on the SNR behavior have led to the need for further investigation.

In this communication, a non-Foster ESA is reported that is based on the near-field resonant parasitic (NFRP) passive and active ESA design technology $[12,22]$. A low-loss non-Foster NIC element is developed and introduced internally into its NFRP element. The experimental verification of the fabricated non-Foster antenna demonstrates that its corresponding passive Chu-limit is surpassed; it attains a measured 5-times enhancement of the $-10-\mathrm{dB}$ fractional bandwidth of the corresponding passive version. it realizes a calculated $-3.52 \mathrm{~dB}$ peak transducer gain and a $44.5 \%$ total efficiency, both of which are significantly higher than other reported designs [20, 23-25]. Because the total equivalent noise of a NIC is proportional to the magnitude of its input impedance [20], the reported low-loss, well-matched design also reduces the amount of inevitable noise. Thus, a SNR improvement is experimentally demonstrated. Details of the effects of the NIC on the radiation performance are explained with analysis and measurements. 


\section{Passive ESA AND the CorResponding NIC Element DesignS}

\section{A. Design of the Passive ESA}

The simple passive NFRP ESA shown in Fig. 1 was selected as the base design. It consists of a coax-fed monopole and a capacitive-loaded loop (CLL) operating as its NFRP radiator [22]. This NFRP design assured a high radiation efficiency, nearly complete impedance matching to the source, and an inherit $\mathrm{dc}$ block characteristic, facilitating its suitability for an internal non-Foster design.

The CLL element shown in Figs. 1 (a) and (b) is printed on a semi-circular, $20 \mathrm{mil}(0.508 \mathrm{~mm})$ thick Rogers Duroid TM 5880 substrate with a $0.017 \mathrm{~mm}$ copper thickness, relative dielectric constant $\varepsilon_{r}=2.2$ and loss tangent $\tan \delta=0.0009$. As illustrated in Fig. 1 (b), one end of the CLL (located along the -y-axis) is connected to the ground plane. The other end (located on the $+y$-axis) has a vertical gap between it and the ground (height $\mathrm{g}_{1}$ ). As shown in Fig. 1(c), the CLL is excited by a monopole printed on the other side of the substrate. The substrate half-disk is centered on and oriented orthogonal to the ground plane, a $2 \mathrm{~mm}$ thick copper ground disk. A second CLL gap (horizontal length $\mathrm{g}_{2}$ ) is located on the upper portion of the CLL in order to explore the frequency-agile characteristics of this NFRP ESA. The case with $\mathrm{g}_{2}=0 \mathrm{~mm}$ acts as the reference case.

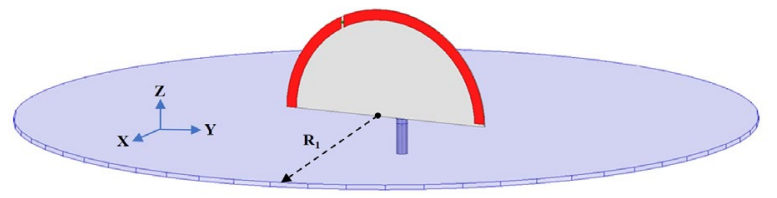

(a)

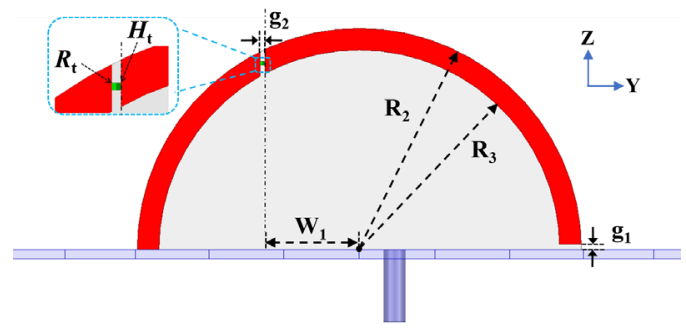

(b)

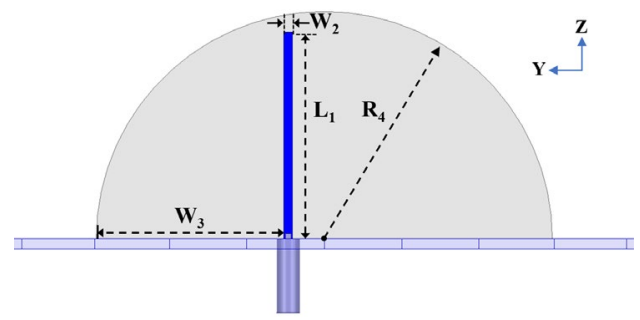

(c)

Fig.1. Passive ESA. (a) 3-D isometric view. (b) Side view of CLL. (c) Side view of feedline. All dimensions are millimeters: $\mathrm{R}_{1}=180, \mathrm{R}_{2}=46, \mathrm{R}_{3}=41.5$, $\mathrm{R}_{4}=46, \mathrm{~L}_{1}=41.8, \mathrm{~W}_{1}=19.5, \mathrm{~W}_{2}=1.8, \mathrm{~W}_{3}=37.8, \mathrm{~g}_{1}=1.1, \mathrm{~g}_{2}=1$.

The ANSYS-ANSOFT high frequency structure simulator (HFSS) was used to simulate the behavior of the ESAs. A pair of lumped element sheets were inserted into the CLL gap $\left(\mathrm{g}_{2}\right)$ and cascaded to represent an inductor $\left(H_{\mathrm{t}}\right)$ in series with a resistor $\left(R_{\mathrm{t}}\right)$. In the limit that the values of both $H_{\mathrm{t}}$ and $R_{\mathrm{t}}$ are zero, this gap is effectively shorted (and, hence, recovers the reference case). This passive ESA has the simulated and measured results shown in Fig. 2. The simulated (measured) resonant frequency is $f_{\text {res }}=455$ (456) $\mathrm{MHz}$ with a narrow fractional bandwidth (FBW-10dB): $0.73 \%(0.77 \%)$. The calculated electrical size at the resonant frequency is $k a=0.44$, where $a$ is the radius of the smallest sphere that completely encloses the radiating element (the ground plane radius $R_{1}$ is much larger and, hence, has little impact on the impedance bandwidth [26]) and can be calculated as the outer radius $R_{2}$ of the CLL element. The Wheel-Chu minimum antenna quality factor corresponding to this $k a$ value is $Q_{\mathrm{Chu}}=(k a)^{-1}+$ $(k a)^{-3}=14.01 \quad[2,4,6]$. The measured value $Q_{\text {meas }}=$ $\mathrm{FBW}^{-1} \times\left(\right.$ VSWR-1)/sqrt $($ VSWR $) \approx 2 /\left(3 \times \mathrm{FBW}_{-10 \mathrm{~dB}}\right)=91.32[2,6,20]$, which confirms that it is far from that lower bound. The simulated peak realized gain is $4.24 \mathrm{dBi}$ with a high radiation efficiency (RE) of 93.7\%.

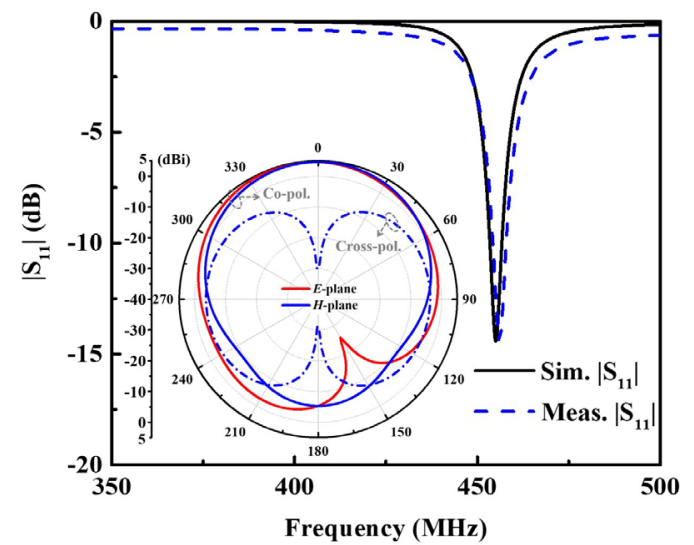

Fig. 2. Simulated and measured $\left|\mathrm{S}_{11}\right|$ values of the passive ESA versus frequency when $g_{2}=0 \mathrm{~mm}$. The simulated realized gain pattern at its resonant frequency $455 \mathrm{MHz}$.

\section{B. Design of NIC}

When the value of $R_{\mathrm{t}}$ is held near zero and the values of $H_{\mathrm{t}}$ are swept from 10 to $28 \mathrm{nH}$ in steps of $2 \mathrm{nH}$, the resonance frequency of the ESA decreases from 424 to $381 \mathrm{MHz}$. This entire range is lower than the reference ESA's value, $455 \mathrm{MHz}$; and as a consequence, a lower $k a$ value is obtained for each case in it. Note that if a capacitor were used rather than an inductor, the resonance frequency range and the associated $k a$ values would be greater than the reference ESA's values.

This frequency-agile behavior is shown in Fig. 3. It provides a simple and effective design bridge between the passive ESA and its desired non-Foster design [12]. The squares mark the resonance frequencies; the dots mark the corresponding reactance values of the input impedance.

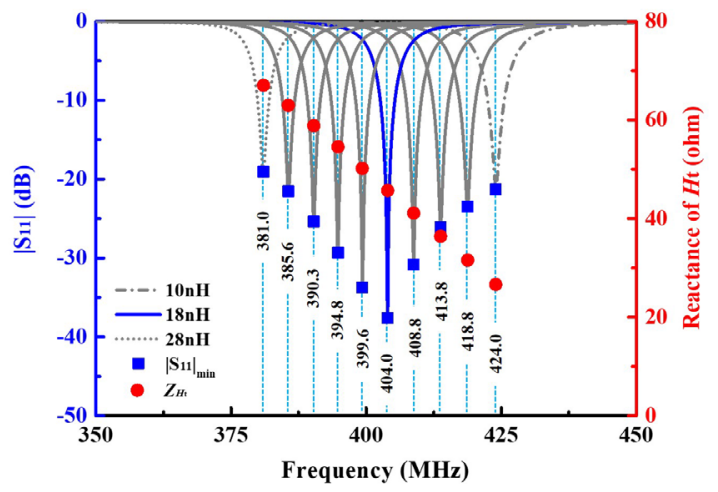

Fig. 3. Simulated frequency-agile performance of the passive ESA.

It is clear that the ESA achieves good impedance matching $\left(\left|\mathrm{S}_{11}\right| \leq\right.$ $-10 \mathrm{~dB}$ ) to its $50 \Omega$ source within its frequency-agile bandwidth. Note 
that the reactance of the inductor $H \mathrm{t}$, which was calculated as $Z_{H_{\mathrm{t}}}=$ $j \times 2 \pi \times f_{\text {res }} \times H_{\mathrm{t}}$, is monotonically decreasing with increasing frequency. Hence, if a non-Foster element is designed to produce a frequency-dependent inductor $H_{\mathrm{t}}$ that matches this behavior, it would transform the frequency-agile bandwidth into an instantaneous bandwidth. Ideally, one would like this to occur with the equivalent resistance of the non-Foster element to be zero to avoid increased losses and, hence, a potentially large decrease in the radiated power.

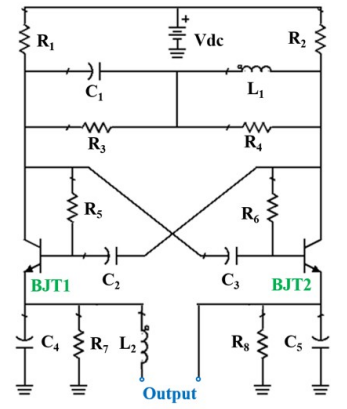

(a)

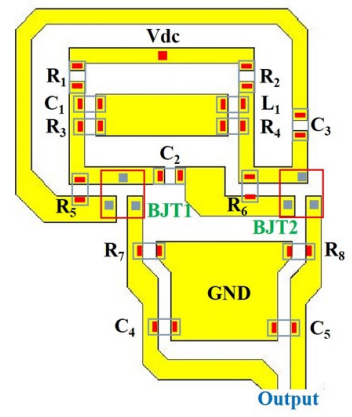

(b)

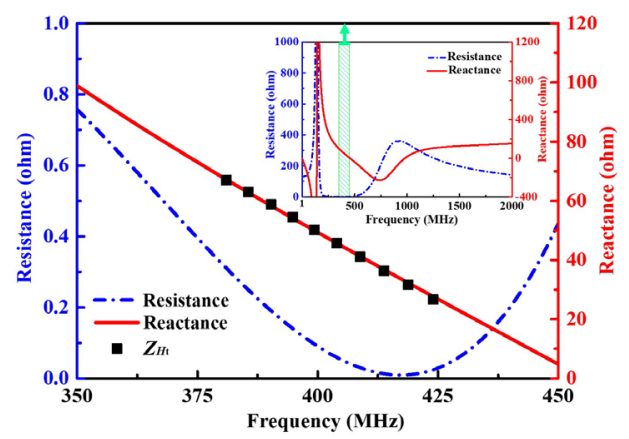

(c)

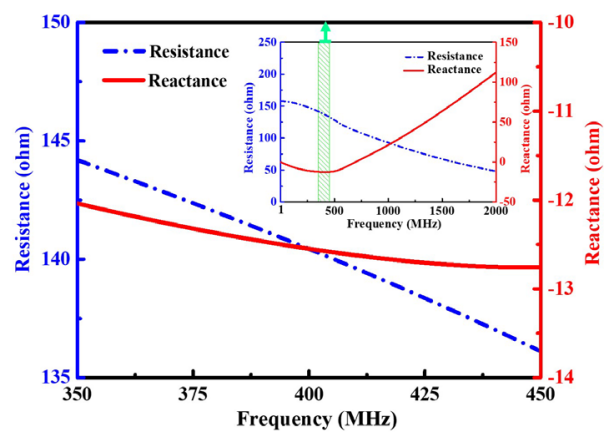

(d)

Fig. 4. Floating one-port NIC element. (a) Schematic circuit with optimized component values: $\mathrm{Vdc}=14 \mathrm{~V}, \mathrm{R}_{1}=917 \Omega, \mathrm{R}_{2}=435 \Omega, \mathrm{R}_{3}=5.9 \mathrm{k} \Omega, \mathrm{R}_{4}=22 \mathrm{k} \Omega$, $\mathrm{R}_{5}=340 \Omega, \mathrm{R}_{6}=1.4 \mathrm{k} \Omega, \mathrm{R}_{7}=36 \Omega, \mathrm{R}_{8}=123 \Omega, \mathrm{L}_{1}=100 \mathrm{nH}, \mathrm{L}_{2}=14 \mathrm{nH}, \mathrm{C}_{1}=1.5 \mathrm{pF}$, $\mathrm{C}_{2}=7.9 \mathrm{pF}, \mathrm{C}_{3}=5.7 \mathrm{pF}, \mathrm{C}_{4}=5 \mathrm{pF}, \mathrm{C}_{5}=0.2 \mathrm{pF}$. The two BJT transistors, BJT1 and BJT2, are both the component number AT41533 from AVAGO. (b) Layout circuit. (c) Simulated output impedance of the NIC schematic and its counterpart with $Z_{H \mathrm{t}}$ when $\mathrm{Vdc}=14 \mathrm{~V}$. (d) Simulated output impedance of the NIC schematic when $\mathrm{Vdc}=0 \mathrm{~V}$.

The equivalent non-Foster impedance required to achieve this goal was extracted from these frequency-agile results. As shown in Fig. 4(a) and (b), the circuit and layout designs of the NIC circuit were simulated with Agilent's advanced design system (ADS). Its output impedance was then assigned to the lumped element sheets $H_{\mathrm{t}}$ and $R_{\mathrm{t}}$ loading the CLL gap $\left(\mathrm{g}_{2}\right)$ in the HFSS co-design simulations [12]. The circuit design is based on the cross-connected two-transistor topology introduced in [9, 12] and belongs to the floating NIC type [11, 19]. Compared to the grounded NIC type, the floating NIC type has a higher sensitivity, but affects smaller changes in the ESA's radiation pattern $[16,27]$. Hence, it is more suitable as the internal non-Foster element. The co-design optimization process was found to be particularly challenging. In fact, this NIC circuit had to be designed with the actual realistic layout shown in Fig. 4(b). If one does not take into account the parasitics associated with all of the transmission lines and lumped elements, instabilities will most likely occur in the assembled system [28].

When the dc source is turned on, i.e., with $\mathrm{Vdc}=14 \mathrm{~V}$, the simulated output impedance of the NIC schematic version is shown in Fig. 4 (c). The values of its imaginary part match their target ones very well. As desired, its real part is small in the desired frequency range and remains positive in higher frequency ranges, e.g., 1 to $2 \mathrm{GHz}$. Again, it is noted that a large positive output resistance would complicate the realization of a matched impedance and it would negatively impact the ESA's radiation performance [23, 29]. On the other hand, a large negative output resistance would lead to an oscillating system, i.e., it would be inherently unstable since the total resistance of the system alone would be negative [30,31]. Consequently, the output resistance of the NIC circuit accounting for the presence of the antenna components was optimized to be as small as possible and non-negative. On the other hand, when the dc source is turned off, i.e., with $\mathrm{Vdc}=0$ $\mathrm{V}$, the simulated output impedance shown in Fig. 4(d) indicates that the resistance of the NIC schematic version becomes very large. This outcome occurs because the resistors $R_{7}$ and $R_{8}$ now have a large impact.

\section{MEASUREMENT OF THE NON-Foster ESA}

This optimized and co-designed NIC circuit was fabricated and integrated into the CLL across the CLL gap $\left(\mathrm{g}_{2}\right)$. The resulting non-Foster ESA was assembled and tested. The prototype and the measurement environment are shown in Fig. 5. Several tunable resistors and capacitors $\left(R_{5}, \ldots, R_{8}\right.$ and $\left.C_{4}, C_{5}\right)$ were introduced into the circuit to provide the ability to fine tune the system (especially the operating point of the transistors, e.g., the value of $\mathrm{Vdc}$ is $14 \mathrm{~V}$ in the simulations while it is $16 \mathrm{~V}$ in the measurements). The experimental verification process included reflection coefficient, radiation performance, and noise behavior measurements.
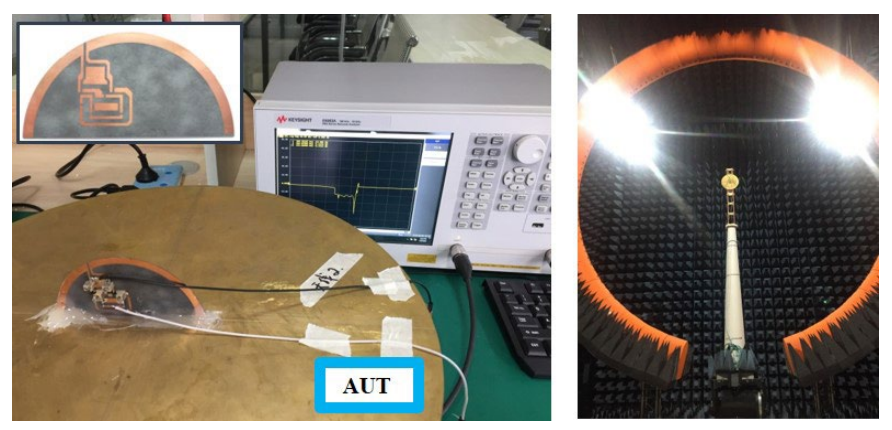

Fig. 5 Photographs of the fabricated non-Foster ESA and its measurement setup. The $\left|\mathrm{S}_{11}\right|$ values were measured with a Keysight E5063A vector network analyzer. The radiation patterns were measured with a SG128 multi-probe antenna measurement system. The final optimized component values were: $\mathrm{Vdc}=16 \mathrm{~V}, \mathrm{R}_{1}=620 \Omega, \mathrm{R}_{2}=510 \Omega, \mathrm{R}_{3}=3.9 \mathrm{k} \Omega, \mathrm{R}_{4}=15 \mathrm{k} \Omega, \mathrm{R}_{5}=1.53 \mathrm{k} \Omega, \mathrm{R}_{6}=3.85$ $\mathrm{k} \Omega, \mathrm{R}_{7}=156 \Omega, \mathrm{R}_{8}=1.04 \mathrm{k} \Omega, \mathrm{L}_{1}=100 \mathrm{nH}, \mathrm{C}_{1}=1.02 \mathrm{pF}, \mathrm{C}_{2}=9.68 \mathrm{pF}, \mathrm{C}_{3}=5.72 \mathrm{pF}$ $\mathrm{C}_{4}=2.23 \mathrm{pF}, \mathrm{C}_{5}=1.59 \mathrm{pF}$. The two BJT transistors, BJT1 and BJT2, are both component number AT41533 from AVAGO. 


\section{A. Reflection Coefficient}

Fig. 6 presents the measured $\left|\mathrm{S}_{11}\right|$ values of the non-Foster ESA when its embedded NIC element is turned on $(\mathrm{Vdc}=16 \mathrm{~V})$ and off $(\mathrm{Vdc}=0 \mathrm{~V})$. They are compared to the passive reference ESA results. It is clear that the NIC decreases the ESA's operational frequency and significantly expands its impedance bandwidth. In particular, the non-Foster ESA was measured to be electrically small with $k a=0.39$ and a -10-dB impedance bandwidth from 403 to $419 \mathrm{MHz}$, i.e., 16 $\mathrm{MHz}$ and $\mathrm{FBW}-10 \mathrm{~dB}=3.9 \%$. In comparison to the measured FBW-10dB $=0.77 \%$ of the passive ESA, a more than 5-times bandwidth enhancement is demonstrated. The Wheel-Chu bound of the quality factor of this system is $Q_{\text {chu }}=19.42$; the measured value $Q_{\text {meas }} \approx$ $2 /(3 \times \mathrm{FBW}-10 \mathrm{~dB})=17.09$. This smaller $\mathrm{Q}$-factor result further demonstrates that the bandwidth of the non-Foster ESA successfully surpassed the basic physics Wheeler-Chu limit $[1,2,6,20]$.

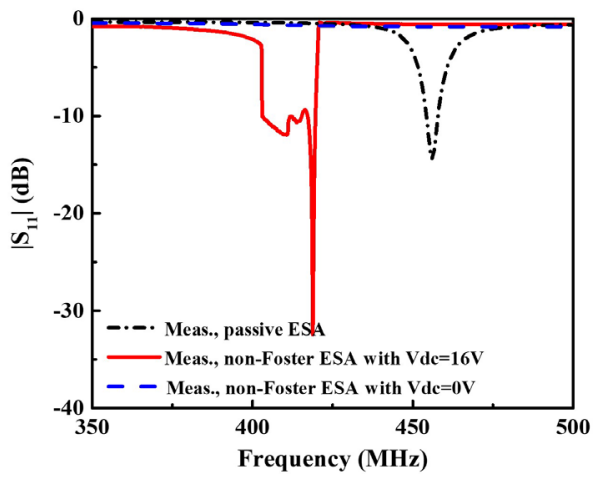

Fig. 6. Measured $\left|S_{11}\right|$ of the passive and non-Foster ESAs versus the source frequency. The results include both non-Foster cases, i.e., when the embedded NIC is turned on and turned off.

\section{B. Radiation performance}

The radiation patterns of the non-Foster ESA shown in Fig. 7 were respectively recorded when the NIC was turned off and on. Compared with the radiation pattern of the passive ESA shown in Fig. 2, the $E$-plane ( $Y O Z$ plane) patterns of the non-Foster ESA are very similar while there are some unexpected ripples in the $H$-plane ( $X O Z$ plane) even when the NIC was turned off. The reason was found to be the orientation of the dc feed lines and the currents on the NIC. When the NIC was turned off, the dc lines play the main role to produce the ripples and increase the cross polarizations. When the NIC was turned on, the additional annular current pathway in the NIC would lead to additional lobes or nulls [23]. Furthermore, a small amount of RF signal may enter into the dc lines and then further enhance the ripple caused by dc lines. Note that, there exist certain ripples in the radiation patterns, whenever the NIC was turned off or on. We should point out that, limited by our measurement facilities, the SG128 multi-probe antenna anechoic chamber, as shown in Fig. 5, has a tall turntable. This caused the usage of dc feed lines with long lengths, which inescapably led to the observed radiation pattern deterioration.

As shown in Fig. 8, the measured peak gain of the non-Foster ESA exhibits reasonable ripples across its -10 - $\mathrm{dB}$ impedance bandwidth ranging from -4.16 to $1.05 \mathrm{dBi}$. When the dc power was turned off, its realized gain was reduced to below $-17 \mathrm{dBi}$. The ESA realizes over 12 $\mathrm{dB}$ of gain improvement when the NIC was turned on.

To better illustrate the merit of designing the NIC to be low-loss, realized gain comparisons are given in Figs. 8 and 9(b). The measured peak realized gain is $1.05 \mathrm{dBi}$ at $405 \mathrm{MHz}$. The peak directivity value, extracted from the HFSS co-simulation results, is $4.57 \mathrm{dBi}$ at $405 \mathrm{MHz}$. There is a slight decrease in the directivity of the non-Foster ESA in the simulation results when compared with its passive reference. The presence of the NIC with its unavoidable resistance loss produces this slight decrease. The calculated transducer gain of our non-Foster ESA is estimated by the ratio of the realized gain to the directivity. It is about $-3.52 \mathrm{~dB}$ and the total efficiency (equal to the transmission efficiency) is $44.5 \%$.

It is important to compare these measured results to related published work. By loading a non-Foster matching network between the ESA and the power source, Refs. [20] and [24] reported a maximum $-4.0 \mathrm{~dB}$ simulated transducer gain (calculated by the ratio of the total radiated power to the input power) with a $-30 \mathrm{dBm}$ input power. By loading an extra amplifier with 4.6-5.7 dB gain between the matching non-Foster matching network and the ESA, an improved simulated peak transducer power gain, about $-4.8 \mathrm{~dB}$, was realized in [25] with a transmission efficiency of $33 \%$. A $-6.88 \mathrm{~dB}$ effective gain was realized in [23] with a total efficiency (calculated as $\eta_{\text {total }}=\left(1-\Gamma_{\text {in }}{ }^{2}\right)$ $\times \mathrm{RE}$ ) of $9.6 \%$ and transducer gain of $-10.18 \mathrm{~dB}$. Consequently, our non-Foster ESA has transducer gain and total efficiency values that are higher than all of these comparison cases. Its better performance benefits from the presence of the effective low-loss NIC, i.e., it has a relatively high measured gain and total efficiency, both of which also facilitate its improved SNR realization.

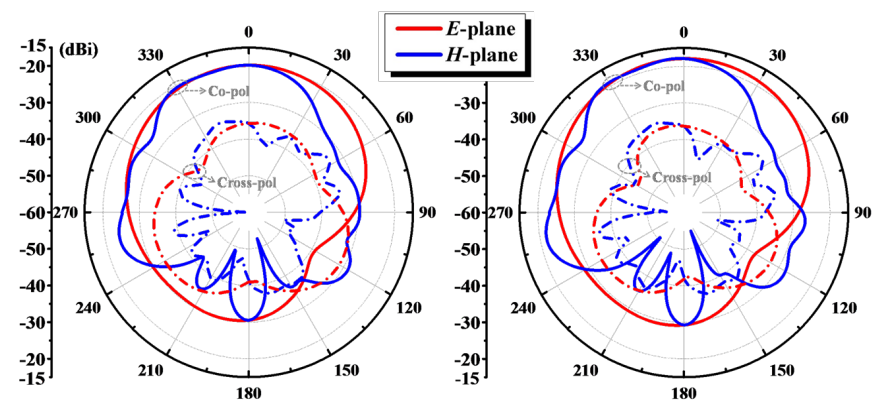

(a)

(b)

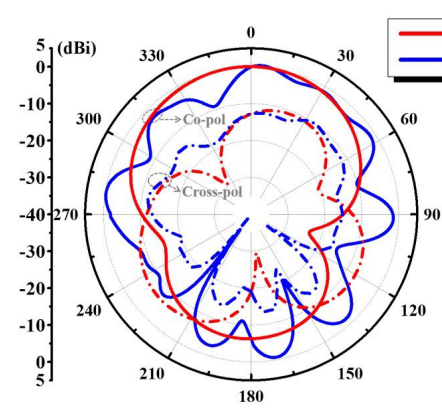

(c)

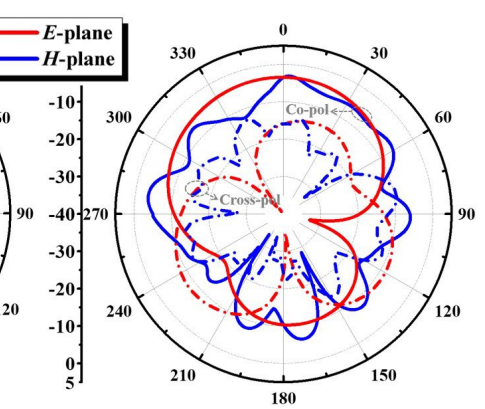

(d)
Fig.7 Measured realized gain patterns of the non-Foster ESA at different frequencies. (a) $403 \mathrm{MHz}$ with the NIC turned off. (b) $419 \mathrm{MHz}$ with the NIC turned off. (c) $403 \mathrm{MHZ}$ with the NIC turned on. (d) $419 \mathrm{MHZ}$ with the NIC turned on. 


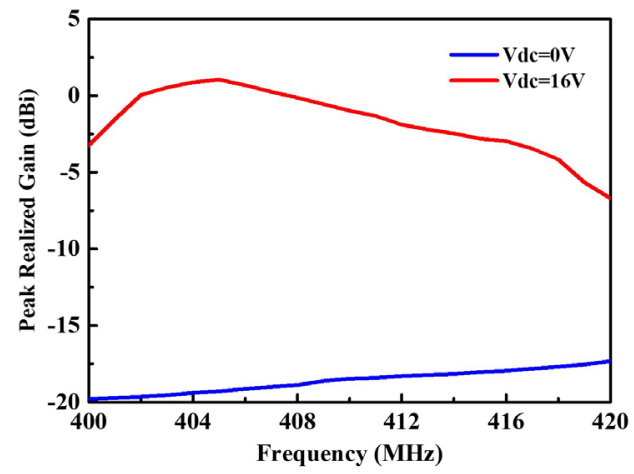

Fig.8 Measured peak realized gain of the non-Foster ESA with the NIC turned off $(\mathrm{Vdc}=0 \mathrm{~V})$ and turned on $(\mathrm{Vdc}=16 \mathrm{~V})$.

\section{Noise and SNR}

A Keysight MXA N9020B spectrum analyzer was used to directly measure the noise spectrum of our non-Foster ESA when the NIC was both turned off and on. The measured results are shown in Fig. 9(a). The total measured noise values for both the $\mathrm{Vdc}=0 \mathrm{~V}$ and $16 \mathrm{~V}$ cases include the environmental noise and the receiver's noise floor. The added noise from the NIC is estimated as the difference between the measured noise for both $\mathrm{Vdc}$ values [20, 25]. Consequently, the calculated SNR (in $\mathrm{dB}$ ) of the non-Foster ESA, which is obtained as the difference between the gain improvement $(\mathrm{dB})$ and the added noise $(\mathrm{dB})$, is also improved, i.e., higher gain and lower noise values are obtained. As shown in Fig. 9(b), the measured results demonstrate that the improvement of the received SNR over the ESA's -10-dB impedance bandwidth when the NIC is turned on is an average of $5 \mathrm{~dB}$ higher than when it is turned off.

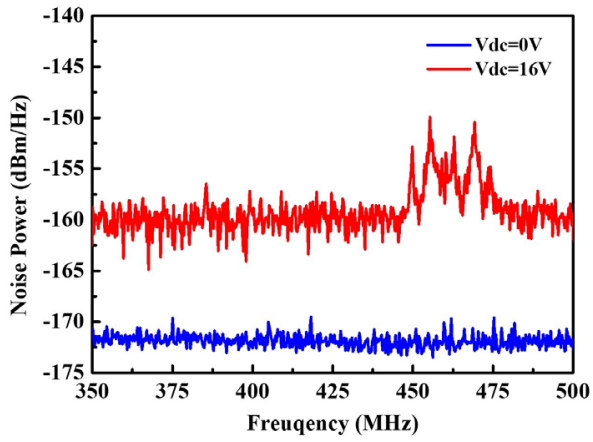

(a)

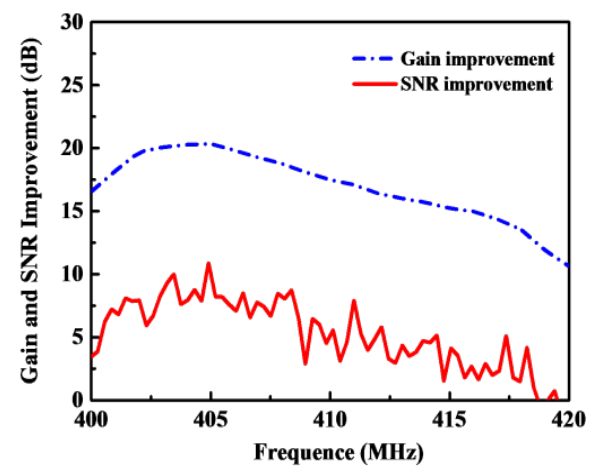

(b)

Fig.9. (a) Measured noise power of the non-Foster ESA with the NIC turned off $(\mathrm{Vdc}=0 \mathrm{~V})$ and turned on $(\mathrm{Vdc}=16 \mathrm{~V})$. (b) Gain and SNR improvements produced by the non-Foster ESA with its NIC turned on compared when it is turned off.

\section{CONCLUSION}

In summary, a NFRP ESA augmented with an internal, low-loss NIC embedded into its NFRP element was experimentally demonstrated. The physics and engineering consequences associated with having a low-loss NIC were emphasized for both its radiation performance and SNR values. Comparisons between the measured results and those obtained with the corresponding passive design confirm that the same electrically-small sized NFRP antenna produced a 5-times expansion of the FBW-10dB fractional bandwidth with a smaller $k a$ value and surpasses the fundamental physics bounds set by the Wheeler-Chu limit for passive systems. Both an average $17 \mathrm{~dB}$ gain and $5 \mathrm{~dB}$ SNR improvements across its operating band suggest that this NIC-augmented NFRP ESA has the potential to meet the needs of emerging $5 \mathrm{G}$ wireless communication systems.

\section{REFERENCES}

[1] A. Erentok and R. W. Ziolkowski, "Metamaterial-inspired efficient electrically small antennas," IEEE Trans. Antennas Propag., vol. 56, no. 3, pp. 691-707, Mar. 2008.

[2] A. D. Yaghjian and S. R. Best, "Impedance, bandwidth, and Q of antennas," IEEE Trans. Antennas Propag., vol. 53, no. 4, pp. 1298-1324, Apr. 2005.

[3] R. M. Fano, "Theoretical limitations of the broadband matching of arbitrary impedances," J. Franklin Instit., vol. 249, pp. 57-83, Jan. 1950, and pp. 139- 155, Feb. 1950.

[4] L. J. Chu, "Physical limitations of omni-directional antennas," J. Appl. Phys., vol. 19, no. 12, pp. 1163-1175, 1948.

[5] H. A. Wheeler, "Fundamental limitations of small antennas," Proc. IRE, vol. 35, pp. 1479-1484, 1947.

[6] D. F. Sievenpiper, D. C. Dawson, M. M. Jacob, T. Kanar, S. Kim, J. Long, and R. G. Quarfoth, "Experimental validation of performance limits and design guidelines for small antennas," IEEE Trans. Antennas Propag., vol. 60, no. 1, pp. 8-19, Jan. 2012.

[7] R. M. Foster, "A reactance theorem," Bell Sys. Tech. J., vol. 3, no. 2, pp. 259-267, Nov. 1924.

[8] J. Linvill, "Transistor Negative-Impedance Converters," Proceedings of the IRE, vol. 41, no. 6, pp. 725-729, Jun. 1953

[9] P. Jin and R. W. Ziolkowski, "Broadband, efficient, electrically small metamaterial-inspired antennas facilitated by active near-field resonant parasitic elements," IEEE Trans. Antennas Propag., vol. 58, no. 2, pp. 318-327, Feb. 2010.

[10] N. Zhu and R. W. Ziolkowski, "Active metamaterial-inspired broad-bandwidth, efficient, electrically small antennas," IEEE Antennas Wireless Propag. Lett., vol. 10, pp. 1582-1585, 2011.

[11] M. Barbuto, A. Monti, F. Bilotti and A. Toscano, "Design of a non-Foster actively loaded SRR and application in metamaterial-inspired components," IEEE Trans. Antennas Propag., vol. 61, no. 3, pp. 1219-1227, Mar. 2013.

[12] M.-C. Tang, T. Shi, and R. W. Ziolkowski, "Electrically small, broadside radiating Huygens source antenna augmented with internal non-Foster elements to increase its bandwidth," IEEE Antennas Wireless Propag. Lett., vol. 16, pp. 712-715, 2017.

[13] J. Church, J.-C. S. Chieh, L. Xu, J. D. Rockway, and D. Arceo, "UHF electrically small box cage loop antenna with an embedded non-Foster load," IEEE Antennas Wireless Propag. Lett., vol. 13, pp. 1329-1332, 2014.

[14] N. Zhu and R. Ziolkowski, "Design and measurements of an electrically small, broad bandwidth, non-Foster circuit-augmented protractor antenna," Appl. Phys. Lett., vol. 101, no. 2, p. 024107, 2012.

[15] C. R. White, J. S. Colburn, and R. D. Nagele, "A non-Foster VHF monopole antenna," IEEE Antennas Wireless Propag. Lett., vol. 11, pp. 584-587, 2012.

[16] H. Mirzaei and G. V. Eleftheriades, "A resonant printed monopole antenna with an embedded non-Foster matching network," IEEE Trans. Antennas Propag., vol. 61, no. 11, pp. 5363-5371, Nov. 2013.

[17] N. Zhu and R. W. Ziolkowski, "Broad bandwidth, electrically small, 
non-Foster element-augmented antenna designs, analyses, and measurements," IEICE Trans. Commun., vol. E96-B, no. 10, pp. 2399-2409, 2013.

[18] G. Fu and S. Sonkusalea, "Broadband wireless radio frequency power telemetry using a metamaterial resonator embedded with non-Foster impedance circuitry," Appl. Phys. Lett., vol. 106, p. 203504, 2015.

[19] S. E. Sussman-Fort and R. M. Rudish, "Non-Foster impedance matching of electrically-small antennas," IEEE Trans. Antennas Propag., vol. 57, no. 8, pp. 2230-2241, Apr. 2009.

[20] M. M. Jacob, and D. F. Sievenpiper, "Gain and noise analysis of non-Foster matched antennas," IEEE Trans. Antennas Propag., vol., 64, no. 12, pp. 4993-5004, Dec. 2016.

[21] Y. Fan, K. Z. Rajab, and Y. Hao, "Noise analysis of broadband active metamaterials with non-Foster loads," J. Appl. Phys., vol. 113, p. 233905, 2013.

[22] M.-C. Tang, R. W. Ziolkowski, S. Xiao, M. Li, and J. Zhang, "Frequency-agile, efficient, near-field resonant parasitic monopole antenna," IEEE Trans. Antennas Propag., vol. 62, no. 3, pp. 1479-1483, Mar. 2014.

[23] F. Albarracín-Vargas, V. Gonzalez-Posadas, F. J. Herraíz-Martinez, and D. Segovia-Vargas, "Design method for actively matched antennas with non-Foster elements," IEEE Trans. Antennas Propag., vol. 64, no. 9, pp. 4118-4123, Sep. 2016.

[24] M. M. Jacob, and D. F. Sievenpiper, "Non-Foster matched antennas for high-power applications," IEEE Trans. Antennas Propag., vol. 65, no. 9, pp. 4461-4469, Sep. 2017.

[25] T.-Y. Shih, and N. Behdad, "Wideband, non-Foster impedance matching of electrically-small transmitting antennas," IEEE Trans. Antennas Propag.,vol. 66, no. 11, pp. 5687 - 5697, Nov. 2018.

[26] R. W. Ziolkowski, "The directivity of a compact antenna: An unforgettable figure of merit," EPJ Appl. Metamat., vol. 4, 7, 2017.

[27] F. Albarracín-Vargas, E. Ugarte-Muñoz, V. González-Posadas and D. Segovia-Vargas, "Sensitivity analysis for active matched antennas with non-Foster elements," IEEE Trans. Antennas Propag., vol. 62, no. 12, pp. 6040-6048, Dec. 2014.

[28] J. Loncar, S. Hrabar, and D. Muha, "Stability of simple lumped-distributed networks with negative capacitors," IEEE Trans. Antennas Propag., vol. 65, no. 1, pp. 390-395, Jan. 2017.

[29] O. O. Tade, P. Gardner and P. S. Hall, "Antenna bandwidth broadening with a negative impedance converter," Int. J. Microw. Wirel. Technol., vol. 5, no. 3, pp. 249-260, Jun. 2013.

[30] E. Ugarte-Muñoz, S. Hrabar, D. Segovia-Vargas, and A. Kiricenko, "Stability of non-Foster reactive elements for use in active metamaterials and antennas," IEEE Trans. Antennas Propag., vol. 60, no. 7, pp. 3490-3494, Jun. 2012.

[31] P.-Y. Chen, C. Argyropoulos and A. Alù, "Broadening the cloaking bandwidth with non-Foster metasurfaces," Phys. Rev. Lett., vol. 111, p. 233001, Dec. 2013. 